\title{
Dynamical Studies of Equations from the Gambier Family
}

Partha GUHA ${ }^{\dagger}$, Anindya GHOSE CHOUDHURY ${ }^{\ddagger}$ and Basil GRAMMATICOS $\S$

$\dagger$ S.N. Bose National Centre for Basic Sciences, JD Block, Sector-3, Salt Lake, Calcutta-700098, India

E-mail: partha@bose.res.in

$\ddagger$ Department of Physics, Surendranath College, 24/2 Mahatma Gandhi Road, Calcutta-700009, India

E-mail: a_ghosechoudhury@rediffmail.com

§ IMNC, Université Paris VII-Paris XI, CNRS, UMR 8165, Bt. 104, 91406 Orsay, France E-mail:grammaticos@univ-paris-diderot.fr

Received December 10, 2010, in final form March 17, 2011; Published online March 22, 2011 doi:10.3842/SIGMA.2011.028

\begin{abstract}
We consider the hierarchy of higher-order Riccati equations and establish their connection with the Gambier equation. Moreover we investigate the relation of equations of the Gambier family to other nonlinear differential systems. In particular we explore their connection to the generalized Ermakov-Pinney and Milne-Pinney equations. In addition we investigate the consequence of introducing Okamoto's folding transformation which maps the reduced Gambier equation to a Liénard type equation. Finally the conjugate Hamiltonian aspects of certain equations belonging to this family and their connection with superintegrability are explored.
\end{abstract}

Key words: Gambier equation; Riccati sequence of differential equations; Milney-Pinney equation; folding transformation; superintegrability

2010 Mathematics Subject Classification: 34C20; 70H05

\section{Introduction}

The Gambier equation plays, for linearizable systems, the same role as the Painlevé VI. Just as one can derive, starting from the latter, all the remaining Painlevé equations through appropriate limiting procedures, in the same way the Gambier equation is a "master" system for the linearizable equations of the Painlevé-Gambier classification [17, 8, 13]. The linearizability of the Gambier equation is based on the fact that it consists in two Riccati equations in cascade (where by "cascade" we mean that the solution of the first Riccati equation is used in order to compute the coefficients of the second one). Thus the Gambier equation, written as a system, has the form:

$$
\begin{aligned}
& \dot{y}=-y^{2}+b y+c, \\
& \dot{x}=a x^{2}+n y x+\sigma .
\end{aligned}
$$

Here $a, b$ and $c$ are functions of the independent variable, $\sigma$ is a constant which can be scaled to 1 unless it happens to be 0 and $n$ is an integer. Eliminating $y$ between (1.1a) and (1.1b) one

\footnotetext{
*This paper is a contribution to the Proceedings of the Conference "Integrable Systems and Geometry" (August 12-17, 2010, Pondicherry University, Puducherry, India). The full collection is available at http://www.emis.de/journals/SIGMA/ISG2010.html
} 
can write the Gambier equation $[10,11]$ as a second-order equation:

$$
\begin{aligned}
\ddot{x}= & \frac{n-1}{n} \frac{\dot{x}^{2}}{x}+a \frac{n+2}{n} x \dot{x}+b \dot{x}-\frac{n-2}{n} \frac{\dot{x}}{x} \sigma-\frac{a^{2}}{n} x^{3} \\
& +(\dot{a}-a b) x^{2}+\left(c n-\frac{2 a \sigma}{n}\right) x-b \sigma-\frac{\sigma^{2}}{n x} .
\end{aligned}
$$

Equation (1.2), although the most general one, is not the only one in the Gambier list. We have in fact

$$
\begin{aligned}
& \ddot{x}=-3 x \dot{x}-x^{3}+q\left(\dot{x}+x^{2}\right), \\
& \ddot{x}=\frac{\dot{x}^{2}}{x}+q \frac{\dot{x}}{x}-\dot{q}+r x \dot{x}+\dot{r} x^{2}, \\
& \ddot{x}=\left(1-\frac{1}{n}\right) \frac{\dot{x}^{2}}{x}+q x \dot{x}-\frac{n q^{2}}{(n+2)^{2}} x^{3}+\frac{n \dot{q}}{n+2} x^{2},
\end{aligned}
$$

where we have preserved the numbering of Gambier [8]. To this list of linearizable systems one must in principle adjoin the equation

$$
\ddot{x}=-2 x \dot{x}+q \dot{x}+\dot{q} x,
$$

which is just the derivative of the Riccati equation.

Starting from the Gambier equation one can obtain almost all the linearizable equations of the Painlevé -Gambier list by the appropriate limits. We start by taking $n=1$ and $\sigma=0$ in (1.2). By the appropriate independent variable transformation and gauge we can put $c=0$ and $a=-1$ leading to (G5). Next we start from (1.2) and take the limit $n \rightarrow \infty$. We find

$$
\ddot{x}=\frac{\dot{x}^{2}}{x}+a x \dot{x}+b \dot{x}-\frac{\dot{x}}{x} \sigma+(\dot{a}-a b) x^{2}+d x-b \sigma
$$

(where we have introduced $d=\lim _{n \rightarrow \infty} c n$ ). Equation (1.3) is not in canonical form. We can reduce it to the standard expression by taking $b=0$ and introducing a gauge $x \rightarrow \rho x$ such that $d=\ddot{\rho} / \rho-\dot{\rho}^{2} / \rho^{2}$. We obtain thus precisely (G13) where $q=1 / \rho$ and $r=a \rho$. Finally we take $\sigma=0$. and perform an independent variable transformation [11] which allows to put $b=0$. It turns out that in this case an additional gauge freedom does exist allowing us to take $c=0$. We obtain thus the equation

$$
\ddot{x}=\frac{n-1}{n} \frac{\dot{x}^{2}}{x}+a \frac{n+2}{n} x \dot{x}-\frac{a^{2}}{n} x^{3}+\dot{a} x^{2},
$$

which is precisely (G14) with $q=a(1+2 / n)$.

The derivative of the Riccati equation, (G6), constitutes an exception in the sense that it cannot be obtained from the Gambier equation. Since we mentioned the derivative of the Riccati it is interesting to point out another "Riccati derivative" which is none other but (1.3) or, in canonical form, (G13). Indeed at the level of the coupled Riccati for the limit $n \rightarrow \infty$ to have a meaning $y$ must go to zero as well. This means that the quadratic term in (1.1a) disappears. Moreover we saw that the canonical form corresponds to $b=0$ and since $c$ is free, we can introduce a new function through $d \equiv n c$. Finally, we divide (1.1b) by $x$ and take the derivative. This leads to the appearance of a term $n \dot{y}$, which from (1.1a) is equal to $d$. Thus, equation (1.4) is a derivative of a Riccati after we have divided by the dependent variable.

At this point one remark is in order concerning the integrability of (1.1). The Gambier equation was first derived by Gambier who complemented the study of Painlevé of second order differential equations the solutions of which have, what is today called, the Painlevé property. 
In order to study the latter in the case of the coupled Riccati system we must start from the observation that since (1.1a) is a Riccati the dominant behaviour of $y$ is $y \sim 1 /\left(z-z_{0}\right)$. An expansion of $y$ around this singularity can be easily established: it involves the functions $b, c$ and their derivatives. Next we turn to (1.1b) which is again a Riccati and thus its singularities are simple poles. However there exist singularities which are due to the singular behaviour of the coefficients, namely $y$. The locations of the singularities of the coefficients of some equation are considered as 'fixed', i.e. not dependent on the initial data. However, in the present case, these singularities are movable because the $z_{0}$ in $y$ is arbitrary and thus should be studied. Because of the pole in $y, x$ has a singular expansion. The expansion has a resonance at position $n-1$ and this introduces a compatibility condition. Thus some constraint on the functions $a, b, c$ must exist for the solutions to have the Painlevé property. However the latter is not necessary when it comes to integrating a linearisable system. The algebraic integrability which stems from the Painlevé property may confer additional "nice" properties to the solutions of the system but, if what we are interested in is just to be able to integrate the system, linearizability suffices. In the case of the Gambier equation this means that if one contents oneself with linearizability no constraints on the free functions do exist and as a matter of fact $n$ does not have to be integer.

\subsection{Motivation and result}

Curiously the Gambier equation has not been the object of many studies. In particular its relation to other integrable differential systems has not been investigated. This is something we intend to remedy in the present paper. We have several results centered around the Gambier equation. These are listed below.

- We explore a connection between the generalized Ermakov-Pinney [5, 18] and the MilnePinney $[16,18]$ equations with the reduced Gambier type equation.

- Using Okamoto's folding transformation we map the reduced Gambier equation to an equation of the Liénard type. We also derive the conjugate Hamiltonian equations for the reduced Gambier equation.

- In addition the conditions under which the reduced Gambier equation becomes a superintegrable system is investigated.

\section{Preliminaries, the generalised Riccati equation}

The properties of higher-order Riccati equations were investigated in [1] where the authors studied in detail the Riccati differential operator,

$$
\mathbb{D}_{R}=\left(\frac{d}{d t}+h x\right)
$$

and the corresponding Riccati differential equations of order $m$ belonging to the hierarchy

$$
\mathbb{D}_{R}^{m} x=0, \quad m=0,1,2, \ldots
$$

Here $h$ is assumed to be a constant independent of $t$. Therefore, as a first generalization one may assume $h$ to be a time-dependent function and define a corresponding differential operator, $\mathbb{T}_{R}$, by

$$
\mathbb{T}_{R}=\left(\frac{d}{d t}+h(t) x\right)
$$


given in [2]. In a manner similar to (2.1) one may define, therefore, a nonautonomous hierarchy of equations by

$$
\mathbb{T}_{R}^{m} x=0, \quad m=0,1,2, \ldots
$$

The first few equations of this hierarchy are given by:

$$
\begin{aligned}
& n=1 \quad \mathbb{T}_{R} x=\left(\frac{d}{d t}+h(t) x\right) x=\dot{x}+h(t) x^{2}, \\
& n=2 \quad \mathbb{T}_{R}^{2} x=\left(\frac{d}{d t}+h(t) x\right)^{2} x=\ddot{x}+3 h(t) x \dot{x}+h^{2}(t) x^{3}+\dot{h}(t) x^{2}, \\
& n=3 \quad \mathbb{T}_{R}^{3} x=\left(\frac{d}{d t}+h(t) x\right)^{3} x=\dddot{x}+4 h(t) x \ddot{x}+6 h^{2}(t) x^{2} \dot{x}+3 h(t) \dot{x}^{2}+h^{3}(t) x^{4} \\
& +5 \dot{h}(t) x \dot{x}+3 h(t) \dot{h}(t) x^{3}+\ddot{h}(t) x^{2},
\end{aligned}
$$

and analogous expressions for higher values of $n$. Obviously the equations become increasingly bulky when $n>3$. Of particular interest is the second member of this family of equations, namely

$$
\ddot{x}+3 h(t) x \dot{x}+\dot{h}(t) x^{2}+h^{2}(t) x^{3}=0 .
$$

Equation (2.2) is called the time-dependent second-order Riccati equation and is known to admit a Lagrangian description [2]. Recently a time-dependent first integral for this equation has been deduced and this has provided the impetus to investigate more deeply the nature of the Riccati operator $\mathbb{T}_{R}$.

\subsection{Linearization of generalised second-order Riccati equations and the Ermakov-Pinney system}

It is well known that the conventional projective transformation

$$
x=\frac{\dot{f}}{h f}
$$

with $h=$ constant can be applied to linearize the time-independent second-order Riccati equation

$$
\ddot{x}+3 h x \dot{x}+h^{2} x^{3}=0 .
$$

When $h=h(t)$ the same projective transformation linearizes the time-dependent second-order Riccati equation (2.2) to the following, namely,

$$
\dddot{f}-\frac{2 \dot{h}}{h} \ddot{f}+\left(2\left(\frac{\dot{h}}{h}\right)^{2}-\frac{\ddot{h}}{h}\right) \dot{f}=0
$$

where $\dot{f}$ denotes differentiation with respect to the argument.

Notice that (2.2) corresponds to the choice $n=1, \sigma=b=0$ and $a=-h$ in (1.2). If $b \neq 0$ then (1.2) reduces (after suitable redefinition) of the time dependent coefficients, namely $b \rightarrow-b, c \rightarrow-c$ and $a=-h$ to

$$
\ddot{x}+3 h x \dot{x}+b \dot{x}+c x+(\dot{h}+b h) x^{2}+h^{2} x^{3}=0 .
$$

This equation has been considered by Sugai [20]. Under the projective transformation defined by $(2.3)$ its corresponding linear version is seen to be

$$
\dddot{f}-\left(\frac{2 \dot{h}}{h}+b\right) \ddot{f}+\left(2\left(\frac{\dot{h}}{h}\right)^{2}-\frac{\ddot{h}}{h}+c-b \frac{\dot{h}}{h}\right) \dot{f}=0 .
$$




\subsubsection{Hill's equation and the Ermakov-Pinney equation}

We wish to recap [12] here the connection between Hill's equation and the Ermakov-Pinney equation, which will be generalized in the next section. It is well known that if $\psi_{1}$ and $\psi_{2}$ are the solutions of the Hill's equation

$$
\Delta \psi=\left(\frac{d^{2}}{d t^{2}}+u(t)\right) \psi=0
$$

then the product $\psi_{i} \psi_{j}(i, j=1,2)$ satisfies the third-order linear equation

$$
\dddot{f}+2 \dot{u} f+4 u \dot{f}=0
$$

and traces out a three dimensional space of solutions. Since the solution of equation (2.4) is spanned by $\operatorname{Span}\left(\psi_{1}^{2}, \psi_{2}^{2}, \psi_{1} \psi_{2}\right)$, its general solution is given by an arbitrary linear combination of the basis vectors, viz

$$
\psi=A \psi_{1}^{2}+2 B \psi_{1} \psi_{2}+C \psi_{2}^{2}
$$

One can show that this is in turn connected with

$$
\ddot{\psi}+u(t) \psi=\frac{\sigma}{\psi^{3}}
$$

where $\sigma$ is a constant.

Proposition 1. If $\psi_{1}$ and $\psi_{2}$ satisfy Hill's equation then

$$
\psi=\sqrt{A \psi_{1}^{2}+2 B \psi_{1} \psi_{2}+C \psi_{2}^{2}}
$$

satisfies the Ermakov equation

$$
\ddot{\psi}+u(t) \psi=\frac{\sigma}{\psi^{3}}, \quad \sigma=A C-B^{2} .
$$

Proof. Since

$$
\psi^{2}=A \psi_{1}^{2}+2 b \psi_{1} \psi_{2}+C \psi_{2}^{2},
$$

differentiation yields

$$
\begin{aligned}
\ddot{\psi}+u(t) \psi= & \frac{1}{\psi^{3}}\left[\left(A \dot{\psi}_{1}^{2}+2 B \dot{\psi}_{1} \dot{\psi}_{2}+C \dot{\psi}_{2}^{2}\right)\left(A \psi_{1}^{2}+2 b \psi_{1} \psi_{2}+C \psi_{2}^{2}\right)\right. \\
& \left.-\left(A \psi_{1} \dot{\psi}_{1}+B\left(\psi_{1} \dot{\psi}_{2}+\dot{\psi}_{1} \psi_{2}\right)+C \psi_{2} \dot{\psi}_{2}\right)^{2}\right]
\end{aligned}
$$

Upon simplification this gives

$$
\ddot{\psi}+u(t) \psi=\frac{A C-B^{2}}{\psi^{3}} W^{2} .
$$

The proof follows by setting $\sigma=A C-B^{2}$ and defining the Wronskian $W=\left(\psi_{1} \dot{\psi}_{2}-\dot{\psi}_{1} \psi_{2}\right)$ to be unity.

It is possible to further extend the above result. 


\subsection{Generalized Ermakov-Pinney system and the Gambier equation}

In this section we extend the previous results. Confining ourselves to a special choice of Hill's equation, viz $u(x)=\Omega^{2}$, a constant, i.e., the harmonic oscillator equation we have the following proposition.

Proposition 2. Let $\psi_{1}$ and $\psi_{2}$ satisfy the equation of a linear harmonic oscillator then

$$
q=\left(A \psi_{1}^{3}+3 B \psi_{1}^{2} \psi_{2}+3 C \psi_{1} \psi_{2}^{2}+D \psi_{2}^{3}\right)^{1 / 3}
$$

satisfies

$$
\ddot{q}+\Omega^{2} q=-\frac{K}{q^{5}}
$$

where $K$ is some constant and $A, B, C, D$ satisfy following conditions

$$
A C-B^{2}=B D-C^{2}=-\Lambda \quad \text { and } \quad A D-B C=0 .
$$

Proof. The proof proceeds in a similar manner as in Proposition 1. Since $q^{3}=A \psi_{1}^{3}+3 B \psi_{1}^{2} \psi_{2}+$ $3 C \psi_{1} \psi_{2}^{2}+D \psi_{2}^{3}$, where $\ddot{\psi}_{i}=-\Omega^{2} \psi_{i}$ for $i=1,2$, upon taking the second derivative we obtain

$$
\begin{aligned}
q^{2} \ddot{q}+2 q \dot{q}^{2}= & -\Omega^{2}\left[A \psi_{1}^{3}+3 B \psi_{1}^{2} \psi_{2}+3 C \psi_{1} \psi_{2}^{2}+D \psi_{2}^{3}\right] \\
& +\left[2 A \psi_{1} \dot{\psi}_{1}^{2}+2 B \dot{\psi}_{1}^{2} \psi_{2}+2 C \dot{\psi}_{2}^{2} \psi_{1}+2 D \dot{\psi}_{2}^{2} \psi_{2}+4 B \psi_{1} \dot{\psi}_{1} \dot{\psi}_{2}+4 C \psi_{2} \dot{\psi}_{1} \dot{\psi}_{2}\right] .
\end{aligned}
$$

This immediately yields

$$
\begin{aligned}
q^{2}\left[\ddot{q}+\Omega^{2} q\right]= & \frac{2}{q^{3}}\left[\left(A \psi_{1}^{3}+3 B \psi_{1}^{2} \psi_{2}+3 C \psi_{1} \psi_{2}^{2}+D \psi_{2}^{3}\right)\right. \\
& \times\left(\left(A \dot{\psi}_{1}^{2}+2 B \dot{\psi}_{1} \dot{\psi}_{2}+C \dot{\psi}_{2}^{2}\right) \psi_{1}+\left(B \dot{\psi}_{1}^{2}+2 C \dot{\psi}_{1} \dot{\psi}_{2}+D \dot{\psi}_{2}^{2}\right) \psi_{2}\right) \\
& \left.-\left(\left(A \psi_{1}^{2}+2 B \psi_{1} \psi_{2}+C \psi_{2}^{2}\right) \dot{\psi}_{2}+\left(B \psi_{1}^{2}+2 C \psi_{1} \psi_{2}+D \psi_{2}^{2}\right) \dot{\psi}_{2}\right)^{2}\right] .
\end{aligned}
$$

Then after a lengthy simplification process we obtain

$$
\begin{aligned}
\ddot{q}+\Omega^{2} q= & \frac{2}{q^{5}}\left[\left(A C-B^{2}\right)\left(\dot{\psi}_{1} \psi_{2}-\psi_{1} \dot{\psi}_{2}\right)^{2} \psi_{1}^{2}+\left(B D-C^{2}\right)\left(\dot{\psi}_{1} \psi_{2}-\psi_{1} \dot{\psi}_{2}\right)^{2} \psi_{2}^{2}\right. \\
& \left.+(A D-B C)\left(\dot{\psi}_{1} \psi_{2}-\psi_{1} \dot{\psi}_{2}\right)^{2} \psi_{1} \psi_{2}\right] \\
= & \frac{2}{q^{5}}\left[\left(A C-B^{2}\right) \psi_{1}^{2}+\left(B D-C^{2}\right) \psi_{2}^{2}+(A D-B C) \psi_{1} \psi_{2}\right] W^{2},
\end{aligned}
$$

where $W=\left(\dot{\psi}_{1} \psi_{2}-\psi_{1} \dot{\psi}_{2}\right)$ is the Wronskian. Let $A C-B^{2}=B D-C^{2}=-\Lambda$ and $A D-B C=0$, then we obtain

$$
\ddot{q}+\Omega^{2} q=-\frac{2 \Lambda W^{2}}{q^{5}}\left(\psi_{1}^{2}+\psi_{2}^{2}\right) .
$$

It is known that for harmonic oscillator $\psi_{1}^{2}+\psi_{2}^{2}=E=$ const. Thus we obtain

$$
\ddot{q}+\Omega^{2} q=-\frac{2 \Lambda E W^{2}}{q^{5}} .
$$

The conditions on the coefficients as stated above imply that $A+C=B+D=0$. 


\subsection{Mapping the Ermakov-Pinney equation to the Gambier equation}

Next we map the generalized Ermakov-Pinney equation to a reduced version of Gambier's equation. Let

$$
x=\frac{a}{q^{3}},
$$

where $a$ is some constant parameter. By a direct computation one can show that if $q$ satisfies equation (2.5) then $x$ satisfies

$$
\ddot{x}-\frac{4}{3} \frac{\dot{x}^{2}}{x}-3 \frac{K}{a^{2}} x^{3}-3 \Omega^{2} x=0 .
$$

Equation (2.6) has a resemblance with the reduced Gambier equation. This can be easily generalized for time-dependent $a(t)$.

Proposition 3. Let $x=\frac{a(t)}{q^{3}}$. If $q$ satisfies the generalized Ermakov-Pinney equation (2.5) then $x$ satisfies

$$
\ddot{x}-\frac{4}{3} \frac{\dot{x}^{2}}{x}-\frac{2}{3} \frac{\dot{a}}{a} \dot{x}-3 \frac{K}{a^{2}} x^{3}+\left(\frac{2}{3}\left(\frac{\dot{a}}{a}\right)^{2}-\frac{\ddot{a}}{a}-3 \Omega^{2}\right) x=0 .
$$

Proof. A direct computation of $(2.5)$ with $q=(a(t) / x)^{1 / 3}$ leads to the proof.

Proposition 4. The equation

$$
\ddot{q}+\lambda \dot{q}+\mu q=-\frac{A}{q^{3}}-\frac{B}{q}
$$

may be mapped to the reduced Gambier equation (1.2) with $n=-2$ and $\sigma=0$ by the following transformation $x=\alpha / q^{2}$, where $\alpha$ is a constant.

Proof. The proof follows a direct computation with $q=\sqrt{\alpha} x^{-1 / 2}$ which gives

$$
\dot{q}=-\frac{\sqrt{\alpha}}{2} x^{-3 / 2} \dot{x}, \quad \ddot{q}=-\frac{\sqrt{\alpha}}{2 x^{3 / 2}}\left[\ddot{x}-\frac{3}{2} \frac{\dot{x}^{2}}{x}\right] .
$$

Substituting these expressions into the above equation we obtain

$$
\ddot{x}=\frac{3}{2} \frac{\dot{x}^{2}}{x}-\lambda \dot{x}+2 \mu x+\frac{2 A}{\alpha^{2}} x^{3}+\frac{2 B}{\alpha} x^{2} .
$$

A comparison allows us to identify the coefficients in terms of those occurring in (1.2) with $n=-2$ and $\sigma=0$.

Note that the transformation $x=\alpha / q^{2}$ is generally referred to as a folding transformation and is discussed in the next section.

\subsection{Folding transformations and the Gambier equation}

This section is devoted to the so called folding transformation of the Gambier equation. Folding transformations were first introduced in [22] by Okamoto, Sakai and Tsuda for the Painlevé equations. According to their terminology folding transformations are algebraic transformations of the Painlevé systems which give rise to a non-trivial quotient map of the space of initial conditions. In a more elementary sense one may say that folding transformations relate the solution of a given Painlevé equation to the square of that of some other equation (which may 
be the same as the initial one) [19]. One of the interesting results of Okamoto et al. was the discovery that $\mathrm{P}_{\mathrm{IV}}$ possesses a folding transformation of degree three, mapping solutions into solutions of the same equation for different values of the parameters.

Let us demonstrate the application of folding transformation for the Gambier equation. When $b=0, n=2$ and $a=$ const, the Gambier equation (1.2) reduces to the following equation

$$
\ddot{x}=\frac{1}{2} \frac{\dot{x}^{2}}{x}+2 a x \dot{x}-\frac{a^{2}}{2} x^{3}-a \sigma x-\frac{\sigma^{2}}{2 x} .
$$

Under the transformation $x=-q^{2}$, this is mapped to the following equation,

$$
\ddot{q}+2 a q^{2} \dot{q}+\left\{\left(\frac{a \sigma}{2}\right) q+\left(\frac{a}{2}\right)^{2} q^{5}+\left(\frac{\sigma}{2}\right)^{2} \frac{1}{q^{3}}\right\}=0,
$$

which is seen to be an equation of the Liénard type. On the other hand if we further set $\sigma=0$ then (2.7) reduces to

$$
\ddot{x}=\frac{1}{2} \frac{\dot{x}^{2}}{x}+2 a x \dot{x}-\frac{a^{2}}{2} x^{3},
$$

and under the same transformation as above we find that

$$
\ddot{q}+2 a q^{2} \dot{q}+\left(\frac{a}{2}\right)^{2} q^{5}=0 .
$$

If however, we set $a=0$ but $\sigma \neq 0$ then (2.7) assumes the following form

$$
\ddot{x}=\frac{\dot{x}^{2}}{2 x}-\frac{\sigma^{2}}{2 x}
$$

which under the transformation $x=-q^{2}$ becomes

$$
\ddot{q}+\frac{\sigma^{2}}{4} \frac{1}{q^{3}}=0 .
$$

\subsection{Generalized Milne-Pinney system and Gambier equation}

The second-order nonlinear differential equation

$$
\ddot{q}+w(t)^{2} \dot{q}+K q^{-5}=0
$$

describes the time evolution of an oscillator with inverse quartic potential. This is a generalization of the Milne-Pinney equation where the evolution is governed by the inverse quadratic potential. It is possible again to map the generalized Milne-Pinney equation to the Gambier equation.

Proposition 5. Let $x=\frac{a(t)}{q^{3}}$. If $q$ satisfies generalized Milne-Pinney equation (2.8) then $x$ satisfies

$$
\ddot{x}-\frac{4}{3} \frac{\dot{x}^{2}}{x}-\left(\frac{2}{3} \frac{\dot{a}}{a}-\frac{w(t)^{2}}{3 a^{2}}\right) \dot{x}-3 \frac{K}{a^{2}} x^{3}+\left(\frac{2}{3}\left(\frac{\dot{a}}{a}\right)^{2}-\frac{\ddot{a}}{a}-\frac{\dot{a}}{3 a^{3}} w(t)^{2}\right) x=0 .
$$

Corollary 1. Let $w(t)^{2}=2 a \dot{a}$. Then the generalized Milne-Pinney equation is mapped to the reduced Gambier equation

$$
\ddot{x}-\frac{4}{3} \frac{\dot{x}^{2}}{x}-\frac{\ddot{a}}{a} x-3 \frac{K}{a^{2}} x^{3}=0 .
$$




\section{Generalized Riccati differential operators and Gambier's equation}

In this section we construct a generalized Riccati differential operator which generates a new sequence of differential equations, that includes the Gambier equation.

We begin by defining the operator

$$
\mathbb{G}:=\left\{\frac{d}{d t}-\frac{n-1}{n} \frac{\dot{x}}{x}-\left(\frac{\sigma}{n x}+\frac{b}{2}+\frac{a x}{n}\right)\right\} .
$$

Here the dot represents differentiation with respect to $t$ and $n$ is an integer. Furthermore $a$ and $b$ are assumed to be functions of $t$ while $\sigma$ is a constant.

Next we consider the sequence of differential equations,

$$
\mathbb{G}^{m} x=0, \quad m=0,1,2, \ldots,
$$

which give rise to the following ODEs:

$$
m=1, \quad \mathbb{G} x=0 \quad \Rightarrow \quad \frac{1}{n} \dot{x}=\frac{\sigma}{n}+\frac{b}{2} x+\frac{a}{n} x^{2},
$$

which is a generalized Riccati equation. For $m=2$ we have $\mathbb{G}^{2} x=0$, which implies

$$
\begin{aligned}
\ddot{x}= & \frac{n-1}{n} \frac{\dot{x}^{2}}{x}+a \frac{n+2}{n} x \dot{x}+b \dot{x}-\frac{n-2}{n} \frac{\dot{x}}{x} \sigma-\frac{a^{2}}{n} x^{3} \\
& +(\dot{a}-a b) x^{2}+\left(c n-\frac{2 a}{n} \sigma\right) x-b \sigma-\frac{\sigma^{2}}{n x},
\end{aligned}
$$

where

$$
c=\frac{\dot{b}}{2}-\frac{b^{2}}{4} .
$$

This procedure can be carried on leading to higher-order equations.

In this paper we will mostly be concerned with (3.2). As has already been mentioned (see also [11]) the linearized family of equations belonging to Gambier's classification can be obtained as canonical reductions of this particular equation.

Indeed for (1.3), viz

$$
\ddot{x}=\frac{n-1}{n} \frac{\dot{x}^{2}}{x}+a \frac{n+2}{n} x \dot{x}-\frac{a^{2}}{n} x^{3}+\dot{a} x^{2},
$$

the corresponding generalized Riccati operator is

$$
\mathbb{G}_{14}=\frac{d}{d t}-\frac{n-1}{n} \frac{\dot{x}}{x}-\frac{a}{n} x .
$$

This corresponds to the operator for the Gambier equation (G14).

On the other hand the choice $n=1, \sigma=0, a=-1$ and $c=0$, so that $\dot{b}=b^{2} / 4$, leads to the fifth equation of Gambier's classification scheme, namely

$$
\ddot{x}=-3 x \dot{x}-x^{3}+b\left(\dot{x}+x^{2}\right),
$$

and corresponds to the following generalized Riccati operator

$$
\mathbb{G}_{5}=\frac{d}{d t}-\frac{b}{2}-x
$$


Taking the limit $n \rightarrow \infty$ in (3.2) we are led to the following equation,

$$
\ddot{x}=\frac{\dot{x}^{2}}{x}+a x \dot{x}+b \dot{x}-\frac{\dot{x}}{x} \sigma+(\dot{a}-a b) x^{2}+d x-b \sigma,
$$

where $d=\lim _{n \rightarrow \infty} n\left(\dot{b} / 2-b^{2} / 4\right)$. However, it is not quite apparent what the corresponding generalized Riccati operator for this equation is, since a direct limiting procedure on (3.1) does not lead to the 13th equation of Gambier's classification.

\subsection{Hamiltonian framework for the Gambier equation (G14)}

We consider equation (1.3)

$$
\ddot{x}=\frac{n-1}{n} \frac{\dot{x}^{2}}{x}+a \frac{n+2}{n} x \dot{x}-\frac{a^{2}}{n} x^{3}+\dot{a} x^{2} .
$$

When $n=-2$ this becomes

$$
\ddot{x}=\frac{3}{2} \frac{\dot{x}^{2}}{x}+\frac{a^{2}}{2} x^{3}+\dot{a} x^{2},
$$

which may be written in the usual manner as the system

$$
\dot{x}=y, \quad \dot{y}=\frac{3}{2} \frac{y^{2}}{x}+\frac{a^{2}}{2} x^{3}+\dot{a} x^{2} .
$$

This system can be expressed in a Hamiltonian form with the following Hamiltonian [3]:

$$
H(x, p, t)=\frac{1}{2} x^{3}(p+b(t))^{2}-\dot{a} \log x,
$$

where $b(t)=\frac{1}{2} \int^{t} a^{2}(s) d s$. The canonical coordinates used are $q=x$ and $p=y x^{-3}-\frac{1}{2} \int^{t} a^{2}(s) d s$. The Hamiltonian equations of motion are

$$
\begin{aligned}
& \dot{x}=\frac{\partial H}{\partial p}=x^{3}(p+b), \\
& \dot{p}=-\frac{\partial H}{\partial x}=-\frac{3}{2} x^{2}(p+b)^{2}+\frac{\dot{a}}{x} .
\end{aligned}
$$

Using $(p+b)=y / x^{3}$ we find that $\dot{x}=y$. On the other hand

$$
\dot{y}=3 x^{2} \dot{x}(p+b)+x^{3}(\dot{p}+\dot{b}) .
$$

Since $\dot{b}=1 / 2 a^{2}$ and $\dot{x}=y$, the last expression can be simplified to yield the second equation in $(3.5)$.

\subsection{Conjugate Hamiltonian equation of Gambier type}

Consider a non-autonomous Hamiltonian system in a $(2+1)$-dimensional extended phase space $\{p, q, t\}$ with a given Hamiltonian $h=H(p, q, t)$. The equation of motion is given by

$$
\frac{d q}{d t}=\frac{\partial H}{\partial p}, \quad \frac{d p}{d t}=-\frac{\partial H}{\partial q} .
$$

The key point is that the Hamiltonian must be explicitly dependent on time which yields $t=$ $T(p, q, h)$. Thus we express

$$
h=H(p, q, T(p, q, h))
$$


and by chain rule we obtain

$$
\frac{\partial T}{\partial p}=-\frac{\partial H}{\partial p}\left(\frac{\partial H}{\partial t}\right)^{-1}, \quad \frac{\partial T}{\partial q}=-\frac{\partial H}{\partial q}\left(\frac{\partial H}{\partial t}\right)^{-1}, \quad \frac{\partial T}{\partial h}=\left(\frac{\partial H}{\partial t}\right)^{-1} .
$$

Since $\frac{d h}{d t}=\frac{\partial H}{\partial t}$ and

$$
\frac{d p}{d h}=\frac{d p}{d t}\left(\frac{\partial H}{\partial t}\right)^{-1}, \quad \frac{d q}{d h}=\frac{d q}{d t}\left(\frac{\partial H}{\partial t}\right)^{-1}, \quad \frac{d t}{d h}=\left(\frac{\partial H}{\partial t}\right)^{-1} .
$$

Substituting (3.1) in (3.3) and considering (3.2) yields

$$
\frac{d p}{d h}=\frac{\partial T}{\partial q}, \quad \frac{d q}{d h}=-\frac{\partial T}{\partial p}, \quad \frac{d t}{d h}=\frac{\partial T}{\partial h} .
$$

This set of equations are known as the conjugate Hamilton's equations.

\subsubsection{The Liénard equation of second type and its conjugate}

The concept of a conjugate Hamiltonian is introduced in [23]. The solution of the equation $h=H(p, x, t)$, where $H$ is a given Hamiltonian containing $t$ explicitly, yields the function $t=T(p, x, h)$. The Hamiltonian system with Hamiltonian $T$ and independent variable $h$ is said to be conjugate to the initial Hamiltonian system with Hamiltonian $H$. Using this construction Fokas and Yang [7] derived the conjugate equation for Painlevé II. In particular, they presented a Lax pair formulation as well as a class of implicit solutions. It may be mentioned here that, in general, a transformation of the hodograph type does not preserve the Painlevé property. We now apply their construction to a Liénard equation of second type.

The Hamiltonian of the Lienard equation of second type, viz, $\ddot{x}+f(x) \dot{x}^{2}+g(x)=0$, may be written as

$$
H(p, x, t)=\frac{1}{2} e^{-2 F(x)}\left(p-g(x) e^{2 F(x)} t\right)^{2}
$$

where $F(x)=\int f(x) d x$. Thus $H=h$ implies

$$
\pm \sqrt{2} e^{2 F(x)}=p-g(x) e^{2 F(x)} t .
$$

It can be easily shown that the dual equations are

$$
\begin{aligned}
& \frac{d x}{d h}=-\frac{\partial T}{\partial p}=-\frac{1}{g(x)} e^{-2 F(x)}, \\
& \frac{d p}{d h}=\frac{\partial T}{\partial x}=\frac{d}{d x}\left(\frac{1}{g(x)} e^{-2 F(x)}\right)\left(p \mp \sqrt{2 h} e^{F(x)}\right)+\frac{1}{g(x)} e^{-2 F(x)}\left(\mp \sqrt{2 h} e^{F(x)} f(x)\right) .
\end{aligned}
$$

Conjugate Hamiltonian for the reduced Gambier equation. The choice

$$
f(x)=-\frac{3}{2 x} \quad \text { and } \quad g(x)=-x^{3}
$$

leads to the conjugate of (3.4) when $a(t)=\sqrt{2}$ as given below

$$
\frac{d x}{d h}=1, \quad \frac{d p}{d h}=\mp \frac{3}{2} \sqrt{2 h} x^{-5 / 2} .
$$

These equations may be solved explicitly yielding

$$
x=\left(h+c_{1}\right), \quad p=\mp \frac{3}{\sqrt{2}} \int \sqrt{\frac{h}{\left(h+c_{1}\right)^{5}}} d h+c_{2},
$$

where $c_{1}$ and $c_{2}$ are arbitrary constants of integrations. 


\section{Generalized Milne-Pinney type equations and superintegrability}

The notion of superintegrability $[6,15]$ has attracted a lot of attention in recent years, due to its various applications and associated mathematical structures. Fris et al. [6], in 1965, studied Euclidean $n=2$ systems which admit separability in two different coordinate systems, and obtained four families of superintegrable potentials with constants of motion linear or quadratic in the velocities (momenta). In this section we focus on a certain reduced version of the Gambier equation (3.2) which exhibits the property of superintegrability. If we set $n=2$ and $a=b=0$ then equation (3.2) reduces to following class of equation

$$
\ddot{x}-\frac{1}{2 x} \dot{x}^{2}+\Omega x-\frac{K}{x}=0,
$$

upon ignoring the constraint on $c$. Our aim is to study such an equation using a singular transformation of Sundman type.

We look for nonlocal transformations, under which a given ordinary differential equation is linearizable. The problem was studied by Duarte et al. [4] by considering basically a transformation of the form

$$
X(T)=F(t, x), \quad d T=G(t, x) d t .
$$

Here $F$ and $G$ are arbitrary smooth functions and it is assumed that the Jacobian $J \equiv \frac{\partial(T, X)}{\partial(t, x)} \neq 0$. If one knows the functional form of $x(t)$, then the latter transformation ceases to be nonlocal. One of the pioneering contributors to this field was K.F. Sundman [21] who introduced the transformation $d t=r d \tau$ in the study of the 3-body problem, where $r$ is the dependent variable (radial component). In particular such transformations are especially effective for the solution of several nonlinear ODEs.

Consider a second-order ordinary differential equation of the Liénard-II type

$$
\ddot{x}+f(x) \dot{x}^{2}+g(x)=0,
$$

and suppose we seek a nonlocal transformation such that it is mapped to the usual equation of a linear harmonic oscillator, namely,

$$
\ddot{X}+\omega^{2} X=0,
$$

(here $\dot{X}=\frac{d X}{d t}$ ) by the nonlocal transformation

$$
\frac{d X}{X}=A(x, t) d x+B(x, t) d t, \quad T=t .
$$

It is a matter of straightforward computation to show that the SODE (4.1) is mapped to (4.2) provided its coefficients satisfy the following conditions:

$$
\begin{aligned}
& A^{2}+A_{x}-A(x) f(x)=0, \\
& B_{x}+2 A(x) B(x)=0, \\
& A(x) g(x)-B^{2}=\omega^{2} .
\end{aligned}
$$

Let $A=v_{x} / v$, then upon solving the first and second equations of the above set we obtain

$$
v_{x}=\exp \left(\int^{x} f(s) d s\right), \quad B(x)=\frac{1}{v^{2}} .
$$


The final equation (4.3) then yields

$$
\frac{v_{x}}{v} g(x)-\frac{1}{v^{4}}=\omega^{2} \text {. }
$$

In particular, let us set $f(x)=\frac{\alpha}{x}$, which readily yields $v=\frac{x^{\alpha+1}}{\alpha+1}(\alpha \neq-1)$. Then $A$ and $B$ are given by the expressions

$$
A(x)=\frac{\alpha+1}{x} \quad \text { and } \quad B(x)=\frac{(\alpha+1)^{2}}{x^{2(\alpha+1)}}
$$

respectively while

$$
g(x)=\frac{(\alpha+1)^{3}}{x^{4 \alpha+3}}+\omega^{2} \frac{x}{(\alpha+1)} .
$$

Therefore, the singular Sundman type transformation is given by

$$
X=x^{\alpha+1} \exp \left((\alpha+1)^{2} \int \frac{d t}{x^{2(\alpha+1)}}\right), \quad T=t,
$$

while the equation assumes the form

$$
\ddot{x}+\frac{\alpha}{x} \dot{x}^{2}+\frac{1}{\alpha+1} \omega^{2} x+\frac{(\alpha+1)^{3}}{x^{4 \alpha+3}}=0 .
$$

Integrating (4.2) we get

$$
\left(\frac{d X}{d T}\right)^{2}+\omega^{2} X^{2}=I(t, x, \dot{x})=\text { const },
$$

where $I(t, x, \dot{x})$ denotes the first integral. Using this recipe we obtain the first integral of (4.4) as

$$
I=(\alpha+1)^{2}\left(x^{\alpha} \dot{x}+\frac{\alpha+1}{x^{\alpha+1}}\right)^{2}+\omega^{2}\left(x^{\alpha+1} \exp \left((\alpha+1)^{2} \int \frac{d t}{x^{2(\alpha+1)}}\right)\right)^{2} .
$$

As shown in [9] (and references therein) the second-order equation (4.1) admits a Lagrangian description via the existence of a Jacobi last multiplier (JLM) $M(x)$, [14] of the form

$$
M(x)=e^{2 F(x)}, \quad \text { where } \quad F(x)=\int^{x} f(s) d s,
$$

with

$$
L(x, \dot{x})=\frac{1}{2} M(x) \dot{x}^{2}-V(x), \quad \text { where the potential is } V(x)=\int^{x} M(s) g(s) d s .
$$

The following proposition is important in identifying superintegrable systems.

Proposition 6. The necessary condition for mapping of $V(x)$ to the superintegrable potential $V(x)=A Q^{2}+B / Q^{2}$ is given by the condition

$$
f(x)=-\frac{5}{4}\left[\frac{g^{\prime \prime}(x)+(f(x) g(x))^{\prime}}{g^{\prime}(x)+f(x) g(x)-2 A}\right]+\frac{g^{\prime \prime \prime}(x)+(f(x) g(x))^{\prime \prime}}{g^{\prime \prime}(x)+(f(x) g(x))^{\prime}},
$$

where $Q=\int^{x} \sqrt{M(s)} d s$ and $A$ and $B$ are arbitrary constants.

Clearly one can directly check that (4.5) is satisfied by (4.4). Moreover the potential is mapped to

$$
V(x)=\int x^{2 \alpha} g(x) d x=\int x^{2 \alpha}\left[\frac{\omega^{2} x}{\alpha+1}+\frac{(\alpha+1)^{3}}{x^{4 \alpha+3}}\right] d x=\frac{1}{2} \frac{\omega^{2}\left(x^{2(\alpha+1)}\right)}{(\alpha+1)^{2}}-\frac{1}{2} \frac{(\alpha+1)^{2}}{x^{2(\alpha+1)}} .
$$

Hence we have $Q=x^{\alpha+1} /(\alpha+1)$ while $A=\omega^{2} / 2$ and $B=-1 / 2$. 


\section{Conclusion}

In this paper we have examined the Gambier equation and its associated systems linking them to several well-known differential equations. In particular we have established the similarity between special cases of the Gambier equation and the generalized Ermakov-Pinney and Milne-Pinney systems. The use of Okamoto's folding transformation leads to a reduction of the Gambier equation to an equation of the Liénard type. In the case of the Gambier equation (G14) it is further observed that the system admits a Hamiltonian description. As a consequence we have studied the conjugate Hamiltonian equations for the second type of Liénard equation which resembles the Gambier equation in certain respects and have indicated their relevance in connection with superintegrability.

Given that the extension of the Gambier system to the discrete case has been already established we could speculate on whether some of the results presented here could be extended to the discrete domain. This is a question we hope to address in some future work of ours.

\section{Acknowledgements}

We wish to thank Alfred Ramani for enlightening discussions and constant encouragements. Two of us (PG and BG) would like to thank K.M. Tamizhmani, organizer of the conference Geometry of Integrable Systems at Pondicherry, India, for the invitation and his kind hospitality. One of the authors (AGC) wishes to acknowledge the support provided by the S.N. Bose National Centre for Basic Sciences, Kolkata in the form of an Associateship.

\section{References}

[1] Cariñena J.F., Guha P., Rañada M., A geometric approach to higher-order Riccati chain: Darboux polynomials and constants of the motion, J. Phys. Conf. Ser. 175 (2009), 012009, 15 pages.

[2] Cariñena J.F., Guha P., Rañada M., Symplectic structure, Darboux functions and higher-order timedependent Riccati equations, unpublished.

[3] Torres del Castillo G.F., The Hamiltonian description of a second-order ODE, J. Phys. A: Math. Theor. 43 (2009), 265202, 9 pages.

[4] Duarte L.G.S., Moreira I.C., Santos F.C., Linearization under non-point transformations, J. Phys. A: Math. Gen. 27 (1994), L739-L743.

[5] Ermakov V.P., Second-order differential equations. Conditions of complete integrability, Univ. Isz. Kiev Series III 20 (1880), no. 9, 1-25 (translation by A.O. Harin).

[6] Fris J., Mandrosov V., Smorodinsky Ya.A., Uhlir M., Winternitz P., On higher symmetries in quantum mechanics, Phys. Lett. 16 (1965), 354-356.

[7] Fokas A.S., Yang D., On a novel class of integrable ODEs related to the Painlevé equations, arXiv:1009.5125.

[8] Gambier B., Sur les équations différentielles du second ordre et du premier degré dont l'intégrale générale est a points critique fixes, Acta Math. 33 (1910), 1-55.

[9] Ghose Choudhury A., Guha P., On isochronous cases of the Cherkas system and Jacobi's last multiplier, J. Phys. A: Math. Theor. 43 (2010), 125202, 12 pages.

[10] Grammaticos B., Ramani A., The Gambier mapping, Phys. A 223 (1996), 125-136, solv-int/9510010.

[11] Grammaticos B., Ramani A., Lafortune S., The Gambier mapping, revisted, Phys. A 253 (1998), 260-270, solv-int/9804011.

[12] Guha P., Stabilizer orbit of Virasoro action and integrable systems, Int. J. Geom. Methods Mod. Phys. 2 (2005), 1-12.

[13] Ince E.L., Ordinary differential equations, Dover Publications, New York, 1944.

[14] Jacobi C.G.J., Sul principio dell'ultimo moltiplicatore, e suo uso come nuovo principio generale di meccanica, Giornale Arcadico di Scienze, Lettere ed Arti 99 (1844), 129-146. 
[15] Makarov A.A., Smorodinsky Ya.A., Valiev Kh., Winternitz P., A systematic search for non-relativistic system with dynamical symmetries, Nuovo Cim. A 52 (1967), 1061-1084.

[16] Milne W.E., The numerical determination of characteristic numbers, Phys. Rev. 35 (1930), 863-867.

[17] Painlevé P., Sur les équations différentielles du second ordre et d'ordre supérieur dont l'intégrale générale est uniforme, Acta Math. 25 (1902), 1-85.

[18] Pinney E., The nonlinear differential equation $y^{\prime \prime}+p(x) y+c y^{-3}=0$, Proc. Amer. Math. Soc. 1 (1950), 681-681.

[19] Ramani A., Grammaticos B., Tamizhmani T., Quadratic relations in continuous and discrete Painlevé equations, J. Phys. A: Math. Gen. 33 (2000), 3033-3044.

[20] Sugai I., Riccati's nonlinear differential equation, Am. Math. Monthly 67 (1960), 134-139.

[21] Sundman K.F., Mémoire sur le problèm des trois corps, Acta Math. 36 (1912), 105-179.

[22] Tsuda T., Okamoto K., Sakai H., Folding transformations of the Painlevé equations, Math. Ann. 331 (2005), 713-738.

[23] Yang D., On conjugate Hamiltonian systems. I. The finite dimensional case, unpublished. 